\title{
Euglycemic Diabetic Ketoacidosis in a Diabetic Patient Treated with SGLT2 Inhibitor
}

\author{
Olga Lavrynenko, MD, PhD ${ }^{1 *}$, Hector Santos, $M D^{1}$, Amando Garza, $M D^{1}$, Rayan Qazi, $M D^{1}$, \\ and Leopoldo Cobos, $M D^{2}$
}

${ }^{1}$ Incarnate Word University/ Laredo Medical Center Laredo, USA

${ }^{2}$ University of Incarnate Word/Laredo Medical Center, Laredo, Texas

\begin{abstract}
Diabetic ketoacidosis (DKA) is a life - threatening complication and must be diagnosed and treated promptly and aggressively. The classic triad of DKA is: Hyperglycemia (blood glucose (BG) $>250 \mathrm{mg} / \mathrm{dl}$ : Anion gap metabolic acidosis $(\mathrm{pH}<7.30$ and bicarbonate $<18 \mathrm{mEq} / \mathrm{L})$ : And ketonemia. With Food and Drug Administration (FDA) approval of the sodium - glucose transporter 2 inhibitors (SGLT2i), DKA can occur with BG levels below $200 \mathrm{mg} / \mathrm{dl}$ and has been defined as euglycemic DKA (EuDKA). Due to the absence of hyperglycemia, the diagnosis of EuDKA is challenging and often delayed.

This 60-year-old diabetic male, treated with empagliflozin and pioglitazone, presented with diarrhea and abdominal pain which started 20 days ago. He was admitted with dehydration and diagnosis of colitis. On admission laboratory evaluation revealed metabolic acidosis with elevated anion gap of $18 \mathrm{mEq} / \mathrm{L}$, bicarbonate of $19 \mathrm{mEq} / \mathrm{L}$, and BG of 146 $\mathrm{mg} / \mathrm{dL}$. There was no history of ingestion of alcohol, salicylates, methanol, ethylene glycol and nothing to suggest lactic acidosis. The plasma creatinine was $0.79 \mathrm{mg} / \mathrm{dl}$. On the following day, he developed an increase in the anion gap to 22 $\mathrm{mEq} / \mathrm{L}$ and further decrease in bicarbonate to $13 \mathrm{mEq} / \mathrm{L}$, and serum ketones were detected. The patient was treated for EuDKA in ICU with intravenous insulin, dextrose (to prevent hypoglycemia), and normal saline with resolution of his symptoms and EuDKA in 3 days.
\end{abstract}

With the widespread use of SGLT2i, physicians need to have a high suspicion of EuDKA in patients who present with an unexplained anion gap acidosis without or only modest elevation in BG concentration

\section{Introduction}

Diabetic ketoacidosis (DKA) is a severe life - threatening complication of diabetes mellitus (DM) and must be diagnosed and treated promptly and aggressively. The classic triad of DKA is: (i) hyperglycemia (blood glucose (BG) more than $250 \mathrm{mg} / \mathrm{dl}$; (ii) anion gap metabolic acidosis ( $\mathrm{pH}$ less than 7.30 and bicarbonate less than $18 \mathrm{mEq} / \mathrm{L}$ ); and (iii) ketonemia [1]. Common presenting features of DKA include polyuria, polydipsia, abdominal pain, nausea, vomiting, weakness, mental status changes, Kussmaul respiration, and fruity breath.

Euglycemic DKA (EuDKA) has been described in diabetic patients with poor oral intake, low carbohydrate/low calorie intake, decreased/missed insulin dose, infections, drug (cocaine) overdose, excessive alcohol consumption, surgical/ traumatic stress [2-9] and recent ly with COVID 19 infection $[10,11]$ (Figure 1). The incidence of EuDKA has increased with introduction of the sodium glucose transporter 2 inhibitors (SGLT2i): Canagliflozin, dapagliflozin, empagliflozin, ertugliflozin, ipragliflozin, luseogliflosin, tofogliflozin, sotogliflozin [6,7-12]. SGLT2 inhibitors are a new class of antihyperglycemic drugs which have gained widespread use for the treatment of patients with type 2 diabetes. They were first marketed in $\mathbf{2 0 1 3}$ for glycemic control and subsequently found to be effective in treating heart failure with reduced ejection fraction (HFrEF) in patients with and without diabetes $[13,14]$, reducing 3-point MACE (nonfatal MI, nonfatal stroke, CV mortality) [15-17], and slowing the progression of diabetic kidney disease $[18,19]$. SGLT2 inhibitors promote osmotic diuresis, reducing preload, and decrease systolic blood pressure, decreasing after load [20,21]. SGLT2i also exert vascular effects, including improved endothelial function and decreased arterial stiffness [20,21]. From the metabolic standpoint, SGLT2i increase plasma glucagon levels and

*Corresponding author: Olga Lavrynenko, Incarnate Word University/ Laredo Medical Center Laredo, USA

Accepted: December 02, 2021

Published online: December 04, 2021

Citation: Lavrynenko O, Santos H, Garza A, et al. (2021) Euglycemic Diabetic Ketoacidosis in a Diabetic Patient Treated with SGLT2 Inhibitor. Clin Diabetes Res 5(1):63-67 
Citation: Lavrynenko O, Santos H, Garza A, et al. (2021) Euglycemic Diabetic Ketoacidosis in a Diabetic Patient Treated with SGLT2 Inhibitor. Clin Diabetes Res 5(1):63-67

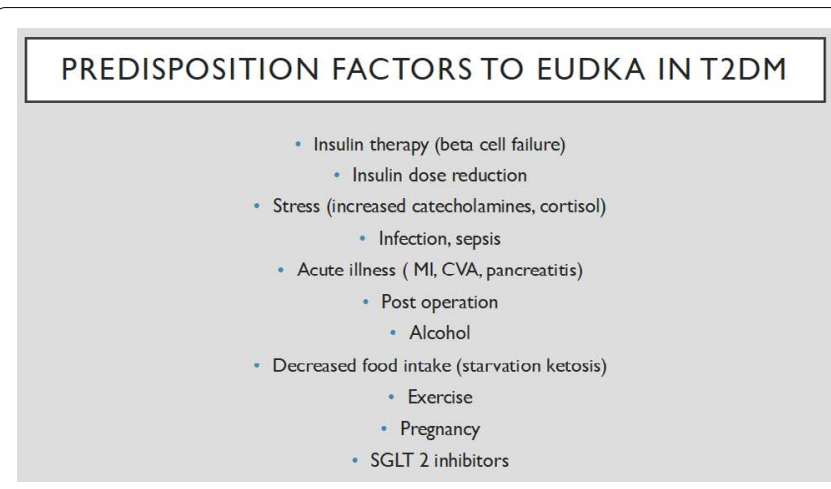

Figure 1. Predisposing factors to EuDKA in type $2 \mathrm{DM}$ patients

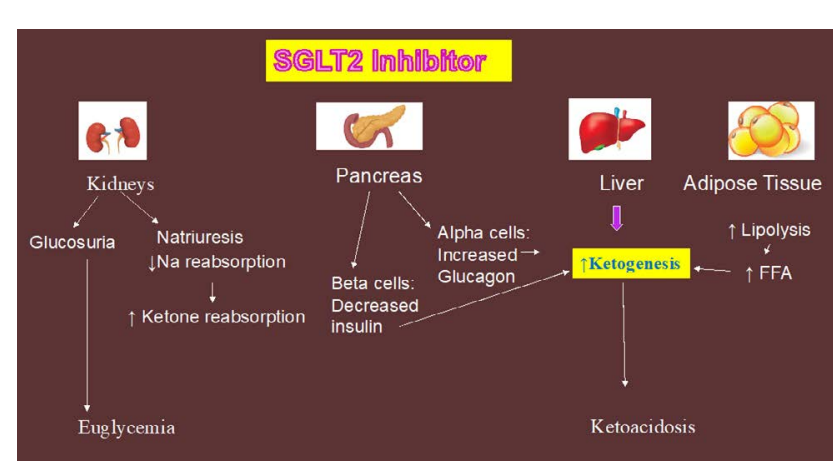

Figure 2: The pathogenesis of EUDKA caused by SGLT2 inhibitors

cause a shift from glucose to fat oxidation [20-22]. In the presence of decreased insulin dose or factors, such as stress, which inhibit endogenous insulin secretion, these changes in substrate (increased fatty acid oxidation) and hormone (increased glucagon) levels can lead to EuDKA (Figure 2).

Due to the atypical presentation of EuDKA without hyperglycemia, patients often do not have polydipsia and polyuria and because of the normal/ near -normal BG level, the diagnosis of EuDKA is challenging and often is delayed.

\section{Case Report}

This case of EuDKA, in a patient taking a SGLT-2 inhibitor (empagliflozin, $25 \mathrm{mg} /$ day), metformin (2000 mg/day) and pioglitazone ( $30 \mathrm{mg} /$ day), was precipitated by dehydration secondary to colitis and decreased fluid intake.

This 60-year-old Hispanic male with diabetes duration of more than 10 years presented to the emergency room with gastrointestinal problems: Diarrhea, bloating, generalized abdominal discomfort and cramps, which started 20 days ago. He described his stools as soft but not watery. He took Imodium as needed and stopped taking his metformin 7 days ago, which provided some improvement. Due to weakness, which the patient attributed to the diarrhea, he began using a walker to ambulate. At the time of admission, the patient could not remember what ant diabetic medications he was taking.

On physical exam, his temperature was $97.6^{\circ} \mathrm{F}$, blood pressure was $123 / 78 \mathrm{mmHg}$, heart rate was 105 beats per minute, and oxygen saturation was $97 \%$ on room air. $\mathrm{He}$ appeared as an ill, middle-aged, no obese male (BMI 26.1 $\mathrm{kg} / \mathrm{m}^{2}$ ) in mild discomfort, with dry mucus membranes and a nontender abdomen without guarding.

On admission laboratory evaluation revealed a metabolic acidosis with an elevated anion gap of $18 \mathrm{mEq} / \mathrm{L}$, bicarbonate of $19 \mathrm{mEq} / \mathrm{L}$, and a serum glucose of $146 \mathrm{mg} / \mathrm{dL}$. Blood gases were not determined on admission because the diagnosis of DKA was not suspected. Plasma chemistry values are shown in Table 1. Urinalysis revealed moderate ketones $(80$ $\mathrm{mg} / \mathrm{dl}$ ) but was otherwise negative. The HbA1c was $7.8 \%$. Blood and stool cultures were negative, including gastrointestinal panel. There was no history of ingestion of alcohol, salicylates, methanol, ethylene glycol and nothing to suggest lactic acidosis.

CT of the abdomen showed (Figure 3) thickening of the wall of the transverse and descending colon along with adjacent inflammation of undetermined etiology including infectious causes, inflammatory bowel disease, or ischemia. ECG was performed (Figure 4) and showed sinus tachycardia, HR 105 bpm, PR interval 126 ms, QRS duration 80 ms, and QT/ QTc 358/473 ms.

He was admitted with dehydration and a presumed diagnosis of colitis of unknown etiology. The patient was treated with metronidazole, levofloxacin and lactate ringer.

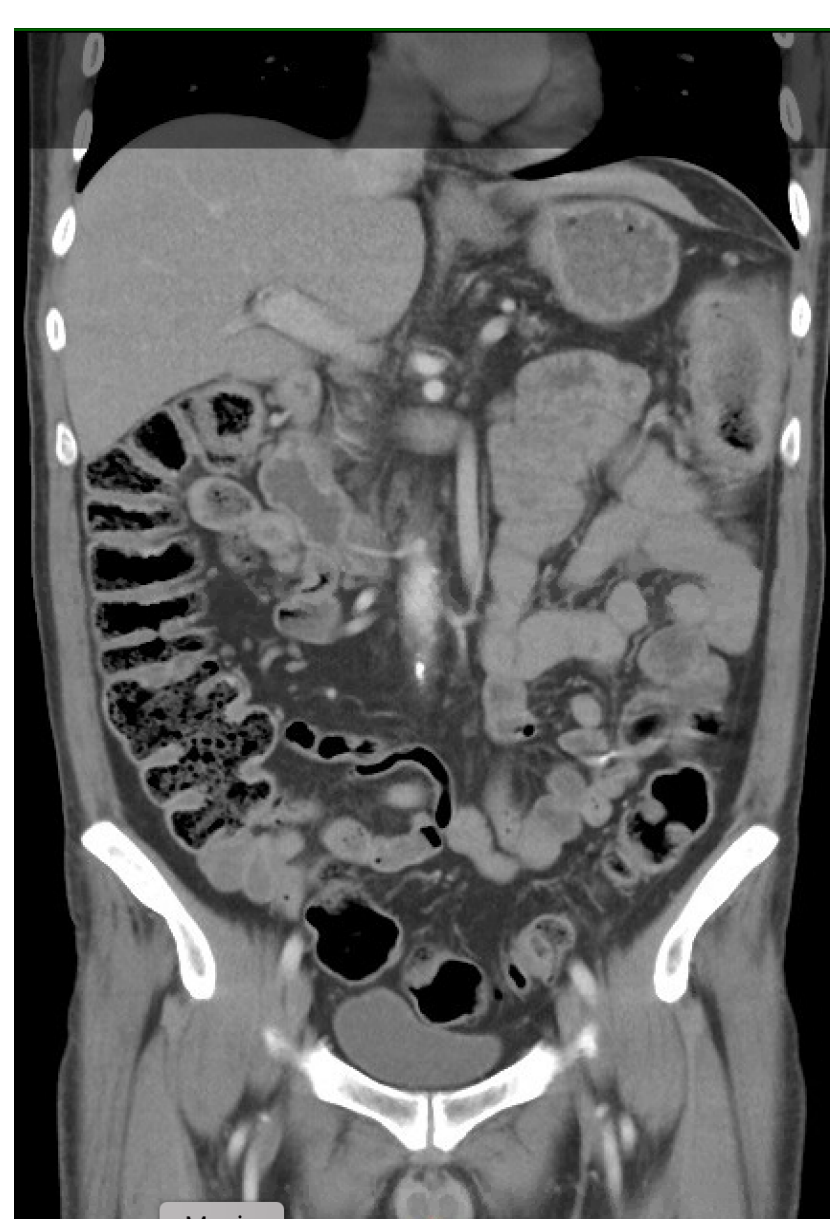

Figure 3: Computer tomography of abdomen with contrast 


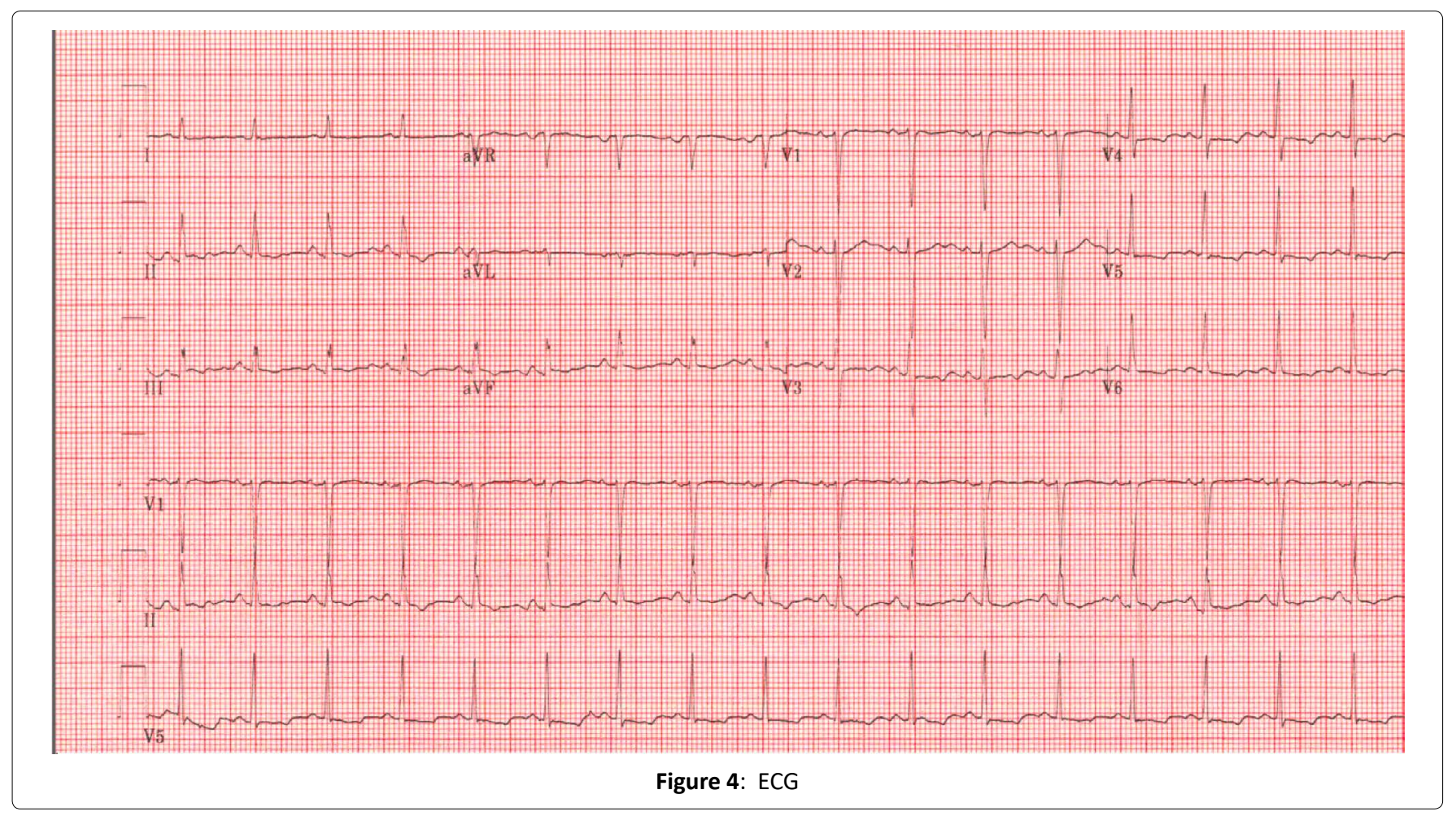

Table 1: Laboratory values before, during, and after treatment

\begin{tabular}{|c|c|c|c|c|c|}
\hline & On admission & During & During treatment & \multirow[t]{2}{*}{ DKA Resolved } & \multirow[t]{2}{*}{ On Discharge } \\
\hline & & \multicolumn{2}{|c|}{ Treatment } & & \\
\hline Glucose, (mg/dl) & 149 & 121 & 172 & 111 & 125 \\
\hline Sodium (mEq/l) & 136 & 135 & 135 & 135 & 138 \\
\hline Chloride (mEq/l) & 99 & 100 & 104 & 109 & 101 \\
\hline Potassium (mEq/l) & 4.6 & 4.8 & 4.3 & 3.7 & 3.4 \\
\hline Anion gap, (mEq/L) & 18 & 22 & 21 & 10 & 6 \\
\hline Bicarbonate, (mEq/L) & 19 & 13 & 10 & 16 & 31 \\
\hline Blood pH & & 7.19 & 7.33 & 7.38 & \\
\hline $\mathrm{PCO} 2(\mathrm{~mm} \mathrm{Hg})$ & & 21.2 & 33.5 & 35.1 & \\
\hline Urine ketones $(\mathrm{mg} / \mathrm{dl})$ & 80 & & & & \\
\hline Serum ketones & & moderate & moderate & small & small \\
\hline Creatinine (mg/dl) & 0.74 & 0.74 & 0.9 & 0.79 & 0.49 \\
\hline Lactate (mmol/L) & 1.8 & 1.7 & & & \\
\hline
\end{tabular}

On the following day, he developed an increase in the anion gap to $22 \mathrm{mEq} / \mathrm{L}$, with a further decrease in the bicarbonate to $13 \mathrm{mEq} / \mathrm{L}$, and ketones were detected in the serum (Table 1). The diagnosis EuDKA was made and the patient was treated with intravenous insulin (6.3 units/hour) and dextrose (to prevent hypoglycemia), with resolution of his symptoms and EuDKA in 3 days. The time course of change in plasma bicarbonate, anion gap, and blood pH is shown in (Table 1).

Latent autoimmune diabetes in adults (LADA) was suspected (DKA in a lean T2DM patient $\left[B M I=21.6 \mathrm{~kg} / \mathrm{m}^{2}\right.$ ], $\mathrm{HbA} 1 \mathrm{c} 7.8 \%$, and $\mathrm{BG}=140-180 \mathrm{mg} / \mathrm{dl})$. GAD antibodies were ordered. Immunology returned as follows: GAD-65 $A B<5$, $I A-2 A B<5.4$, insulin $A B<0.4$, demonstrating that LADA was not present.

The patient was discharged without symptoms on metformin with discontinuation of the SGLT2 inhibitor and follow up appointment in the endocrinology clinic.

\section{Discussion}

Empagliflozin is a SGLT2 inhibitor used for the treatment of type 2 DM and has been shown to decrease the MACE endpoint [15-17], reduce hospitalization for heart failure in patients with reduced ejection fraction $[13,14]$, and slow the progression of diabetic kidney disease $[18,19]$. As with other SGLT2 inhibitors, empagliflozin works by reversibly inhibiting the sodium glucose co transporter 2 located in the proximal renal tubule, thereby reducing the reabsorption of filtered glucose and promoting glucosuria [20,21,23]. Specifically, the SGLT2i lower the renal threshold for glucose below the fasting plasma glucose concentration observed in no diabetic individuals, explaining their marked glucosuria effect $[20,21,23]$. Additionally, SGLT2 inhibitors stimulate the release of glucagon and increase lipolysis in adipose tissue leading to an increase in circulating plasma free fatty acid (FFA) levels $[20,21,24]$. FFA are the precursors for ketogenesis 
and glucagon stimulates ketogenesis in the liver $[20,21]$. Further, as the plasma glucose concentration declines, the plasma insulin concentration decreases, further promoting hepatic ketogenesis.

All members of the SGLT2i class have been associated with cases of EUDKA, and the FDA has revised the SGLT2 $i$ labels to include a warning for the increased risk of DKA in T2DM patients $[25,26]$. However, the American Association of Clinical Endocrinologists announced that evidence points to a low risk of DKA with these medications and concluded that their benefits far outweigh any risk [27].

Many of the patients in the reported cases of EuDKA with SGLT2i were consuming a low caloric intake (ketogenic diet) or had discontinued insulin [2-9]. Episodes also can be triggered by surgery, pregnancy, infection, trauma, any major illness, nausea/ vomiting with reduced food intake, and excessive alcohol intake [2-9].

An important and less well-appreciated cause of EuDKA is intravascular volume depletion. In an elegant study performed by Perry et al. [28] in both healthy no diabetic and type 2 diabetic rats, dapagliflozin in the presence of insulinopenia and volume depletion, (leading to elevated plasma catecholamine's and corticosterone concentrations) promoted ketogenesis. These hemodynamic and hormonal derangements stimulated adipose tissue lipolysis, hepatic acetyl-CoA content, and hepatic ketogenesis. Volume depletion was necessary but not sufficient to promote DKA. Similarly, insulinopenia was necessary but not sufficient to promote DKA. In the present case, the patient was markedly volume depleted on admission and, although not measured, most likely was insulin deficient based upon the natural history of T2DM [29]. This path physiologic sequence emphasizes the importance of volume depletion in the pathogenesis of EuDKA and the need for aggressive normal saline replacement to reverse the DKA.

The signs and symptoms of EuDKA will vary depending on the underlying precipitating cause, but the laboratory findings will be similar to those of typical hyperglycaemic DKA with the exception of the normal/ mildly elevated blood glucose concentration. Polyuria, polydipsia, and mental status changes often are not present.

The diagnosis of EuDKA should be considered in any diabetic patient who presents with an unexplained anion gap and metabolic acidosis irrespective of the plasma glucose concentration, and should be confirmed by measuring the plasma ketone concentration.

\section{Conclusion}

In our patient, the diagnosis of EuDKA was delayed because the BG was only $149 \mathrm{mg} / \mathrm{dL}$, and the significance of the ketonuria was not appreciated. His anion gap initially was attributed to starvation ketosis and the patient was admitted to a regular, non-ICU floor.

With the widespread use of SGLT2 inhibitors, physicians need to have a high suspicion of EuDKA in patients who present with an unexplained anion gap acidosis without significant elevation in the blood glucose concentration.
In patients with T2DM who are taking a SGLT-2 inhibitor and/or who are consuming a low calorie, carbohydrate restricted diet and who present with nausea/vomiting, fatigue and anion gap metabolic acidosis, measuring the serum ketones is critical in order not to miss the diagnosis of EuDKA.

Treatment of EuDKA requires aggressive volume replacement with normal saline (with potassium as indicated) and insulin plus dextrose, due to the low serum blood glucose. IV insulin therapy must be continued until the ketosis resolves and the amino gap closes. Insulin infusion cannot be stopped because of a normal blood glucose level.

\section{References}

1. Kitabchi AE, Umpierrez GE, Miles JM, et al. (2009) Hyperglycaemic crisis in adults with diabetes. Diabetes Care 32: 1335-1343.

2. Anne Peters, Elizabeth Bushur, John Buse, et al. (2015) Euglycemic Diabetic Ketoacidosis: A Potential Complication of Treatment with Sodium-Glucose Co transporter 2 Inhibition. Diabetes Care 38: 1687 -1693.

3. Hine J (2015) SGLT Inhibition and Euglycemic Diabetic Ketoacidosis. Lancet Diabetes Endocrinal 3: 503 - 504.

4. Tomohide Hayam, Yoshiro Kato, Hayami T et al. (2015) Case of Ketoacidosis by a Sodium-Glucose Co transporter 2 Inhibitor in a Diabetic Patient with a Low-Carbohydrate Diet. J Diabetes Investig 6: 587-590.

5. Brit Long, Skyler Lentz, Alex Koyfman, et al. (2021) Euglycemic diabetic ketoacidosis: Etiologies, evaluation, and management. American Journal of Emergency Medicine 44: 157-160.

6. Liu J, Li L, Li S, et al. (2020) Sodium-glucose co-transporter-2 inhibitors and the risk of diabetic ketoacidosis in patients with type 2 diabetes: A systematic review and meta-analysis of randomized controlled trials. Diabetes Obes Metab 22: 1619

7. Ueda P, Svanström H, Melbye M, et al. (2018) Sodium glucose co transporter 2 inhibitors and risk of serious adverse events: nationwide register based cohort study.

8. Ahmad Yehya, Archana Sadhu (2020) Sodium-Glucose Co transporter 2 Inhibitor-Associated Prolonged Euglycemic Diabetic Ketoacidosis in Type 2 Diabetes: A Case Report and Literature Review Clin Diabetes 38 : 112-116.

9. Serena Mistry, Deidre Cocks Eshler (2021) Euglycemic diabetic ketoacidosis caused by SGLT2 inhibitors and ketogenic diet; a case series and review of literature. AACE Clinical Case Rep 7: 17-19.

10. Oriot P, Hermans MP (2020) Euglecimic diabetic Ketoacidosis in a patient with type 1 diabetes and SARS-CoV-2 pneumonia: Case report and review of the literature Acta Clin Belg 16: 1-5.

11. Bhagwan Dass, Andrew Beck, Cody Holmses, et al. (2021) Euglycemic DKA (euDKA) as a presentation of COVID-19 Clin Case Rep 9: 395-398.

12. Julio Rosenstock, Ele Ferrannini (2015) Euglycemic diabetic ketoacidosis: a predictable, detectable, and preventable safety concern with SGLT2 Inhibitors. Diabetes Care 38: 1638-1642.

13. McMurray JJV, Solomon SD, Inzucchi SE, et al. (2019) Committees D-HT, Investigators. Dapagliflozin in Patients with Heart Failure and Reduced Ejection Fraction. N Engl J Med 381: 1995-2008. 
14. Packer M, Anker SD, Butler J, et al. (2020) Investigators EM-RT. Cardiovascular and Renal Outcomes with Empagliflozin in Heart Failure. N Engl J Med 383: 1413-1424.

15. Zinman B, Wanner C, Lachin J M et al. (2015) Empagliflozin, cardiovascular outcomes, and mortality in type 2 diabetes. $\mathrm{N}$ Engl J Med 373: 2117-2128.

16. Neal B, Perkovic V, Mahaffey KW, et al. (2017) Canagliflozin and Cardiovascular and Renal Events in Type 2 Diabetes. N Engl J Med 377: 644-657.

17. Wiviott SD, Raz I, Bonaca MP, et al. (2019) Dapagliflozin and Cardiovascular Outcomes in Type 2 Diabetes. N Engl J Med 380: 347-357.

18. Heerspink HJL, Stefansson BV, Correa-Rotter R, et al. (2020) Dapagliflozin in Patients with Chronic Kidney Disease. N Engl J Med 383: 1436-1446.

19. Perkovic V, Jardine MJ, Neal B, et al. (2019) Canagliflozin and Renal Outcomes in Type 2 Diabetes and Nephropathy. N Engl J Med 380: 2295-2306.

20. Abdul-Ghani MA, Norton L, DeFronzo RA et al. (2015) Renal sodium-glucose co transporter inhibition in the management of type 2 diabetes mellitus. Am J Physiol Renal Physiol 309: F889-900.

21. DeFronzo RA, Norton L, Abdul-Ghani M et al. (2017) Renal, metabolic and cardiovascular considerations of SGLT2 inhibition. Nat Rev Nephrol 13: 11-26.
22. Merovci A, Solis-Herrera C, Daniele G, et al. (2014) Dapagliflozin improves muscle insulin sensitivity but enhances endogenous glucose production. J Clin Invest 124: 509-514.

23. DeFronzo RA, Hompesch M, Kasichayanula S, et al. (2013) Characterization of renal glucose reabsorption in response to dapagliflozin in healthy subjects and subjects with type 2 diabetes. Diabetes Care 36: 3169-3176.

24. Ferrannini E, Muscelli E, Frascerra S, et al. (2014) Metabolic response to sodium-glucose co transporter 2 inhibition in type 2 diabetic patients. J Clin Invest 124: 499-508.

25. (2015) FDA. FDA warns that SGLT2 inhibitors for diabetes may result in a serious condition of too much acid in the blood.

26. (2015) FDA. FDA Drug Safety Communication: FDA revises labels of SGLT2 inhibitors for diabetes to include warnings about too much acid in the blood and serious urinary tract infections.

27. AACE/ACE Scientific and Clinical Review: Association of SGLT2i and DKA.

28. Rachel J. Perry, Aviva Rabin-Court, et al. 2019) Dehydration and insulinopenia are necessary and sufficient for euglycemic ketoacidosis in SGLT2 inhibitor-treated rats. Nature Communications 10: 548 .

29. DeFronzo RA (2009) From the triumvirate to the ominous octet: A new paradigm for the treatment of type 2 diabetes mellitus. Diabetes 58: 773-795. 